\title{
Pion electromagnetic form factor from full lattice QCD
}

\section{HPQCD Collaboration}

Jonna Koponen*

SUPA, School of Physics and Astronomy, University of Glasgow, Glasgow, G12 8QQ, UK

E-mail: jonna.koponen@glasgow.ac.uk

\section{Francis Bursa}

SUPA, School of Physics and Astronomy, University of Glasgow, Glasgow, G12 8QQ, UK

\section{Christine Davies}

SUPA, School of Physics and Astronomy, University of Glasgow, Glasgow, G12 8QQ, UK

\section{Gordon Donald}

SUPA, School of Physics and Astronomy, University of Glasgow, Glasgow, G12 8QQ, UK

School of Mathematics, Trinity College, Dublin 2, Ireland

\section{Rachel Dowdall}

DAMTP, University of Cambridge, Wilberforce Road, Cambridge CB3 OWA, UK

\begin{abstract}
We present preliminary results from the first calculation of the pion electromagnetic form factor at physical light quark masses. This form factor parameterises the deviations from the behaviour of a point-like particle when a photon hits the pion. These deviations result from the internal structure of the pion and can thus be calculated in QCD. We use three sets (different lattice spacings) of $n_{f}=2+1+1$ lattice configurations generated by the MILC collaboration. The Highly Improved Staggered Quark formalism (HISQ) is used for all of the sea and valence quarks. Using lattice configurations with $u / d$ quark masses very close to the physical value is an advantage, as we avoid the chiral extrapolation. We study the shape of the vector $\left(f_{+}\right)$form factor in the $q^{2}$ range from 0 to $-0.12 \mathrm{GeV}^{2}$ and extract the mean square radius, $\left\langle r_{v}^{2}\right\rangle$. The shape of the vector form factor and the resulting radius is compared with experiment.
\end{abstract}

31st International Symposium on Lattice Field Theory LATTICE 2013

July 29 - August 3, 2013

Mainz, Germany

\footnotetext{
*Speaker.
} 


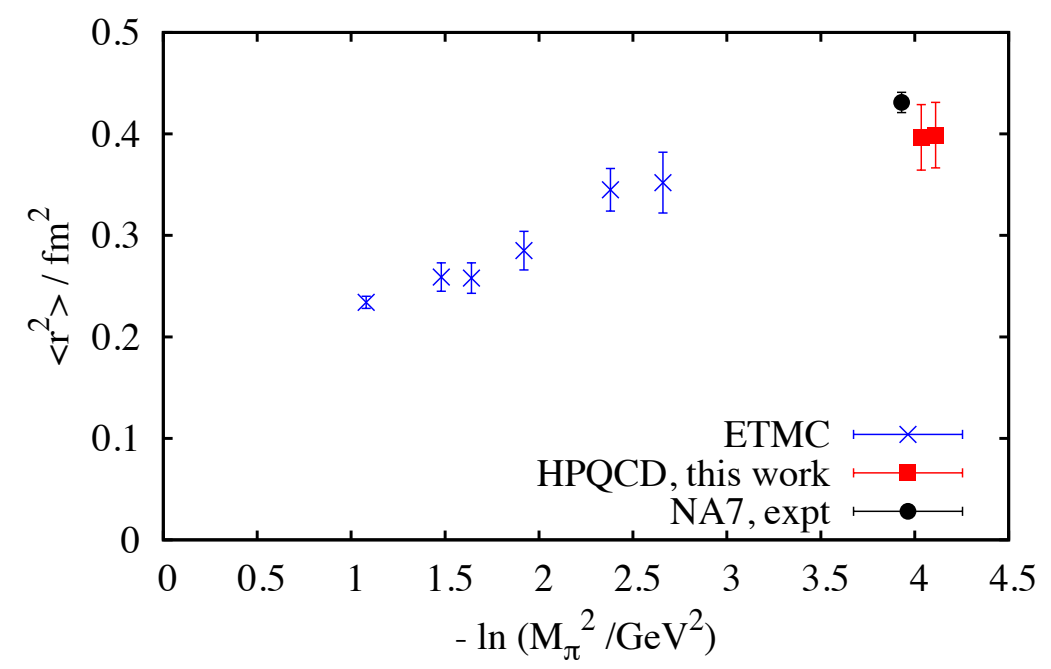

Figure 1: Dependence of the charge mean square radius on the pion mass. Note that we plot the radius as a function of $\ln \left(m_{\pi}^{2}\right)$, not $m_{\pi}^{2}$. The experimental result is from [3] and the ETMC lattice results are from [1].

\section{Motivation}

The electromagnetic form factor of the charged $\pi$ meson parameterises the deviations from the behaviour of a point-like particle when struck by a photon. These deviations arise from the internal structure of the pion: constituent quarks and their strong interaction. The form factor can be calculated in Lattice QCD, but it is desirable to work at the physical pion mass to avoid chiral extrapolation. Plots of lattice determinations of the pion charge radius as a function of pion mass - like Fig. 1, or Fig. 11 in [1] or Fig. 4 in the very recent review paper [2] - show very clearly that the extrapolation to physical pion mass plays a key role, if the pion masses one works at are much heavier than the physical mass. On the experimental side, the vector form factor has been measured by NA7 collaboration [3] in a $\pi-e$ scattering experiment, which allows a comparison between theory and experiment.

\section{Lattice configurations}

We use the $n_{f}=2+1+1$ HISQ (Highly Improved Staggered Quark) physical pion mass lattice configurations provided by MILC Collaboration [4]. Three ensembles (different lattice spacings) are used in this study - see details in Table 1. We use the HISQ action for valence quarks as well, and using the same light quark mass as in the sea. We have $L m_{\pi} \approx 4$ for the coarse $(a=0.12 \mathrm{fm})$ and fine $(a=0.088 \mathrm{fm})$ lattices, so finite volume effects are expected to be very small. We have good statistics with 1000 configurations (for the very coarse and coarse ensembles) and four time sources per configuration.

\section{Form Factors}

On the lattice, form factors are extracted from 3-point correlators - see Fig. 2. We have two pseudoscalar mesons, the initial and final state pions, time $T$ apart, and we use a 1-link spatial 


\begin{tabular}{|c|c|c|c|c|c|c|c|c|}
\hline \hline Set & $a / \mathrm{fm}$ & $a m_{l}$ & $a m_{s}$ & $a m_{c}$ & $m_{\pi} / \mathrm{MeV}$ & $(L / a)^{3} \times L_{t} / a$ & $N_{\text {conf }}$ & $T / a$ \\
\hline 1 & 0.15 & 0.00235 & 0.0647 & 0.831 & 133 & $32^{3} \times 48$ & 1000 & $9,12,15$ \\
2 & 0.12 & 0.00184 & 0.0507 & 0.628 & 133 & $48^{3} \times 64$ & 1000 & $12,15,18$ \\
3 & 0.088 & 0.00120 & 0.0363 & 0.432 & 128 & $64^{3} \times 96$ & 223 & $16,21,26$ \\
\hline \hline
\end{tabular}

Table 1: Details of the MILC $2+1+1$ flavor lattice configurations used in this study. Set 1 is very coarse, set 2 is coarse and set 3 is fine ensemble. The second column is the lattice spacing ([5], using $w_{0}$ to determine the scale) and columns 3-6 list the sea quark masses and the pion mass. Columns 7 and 8 give the size of the lattice and the number of configurations used. Four time sources are used per configuration to get good statistics. $T$ in the 9th column is the separation between the initial and final state mesons. We use multiple values of $T$ to improve extraction of the ground state matrix elements.
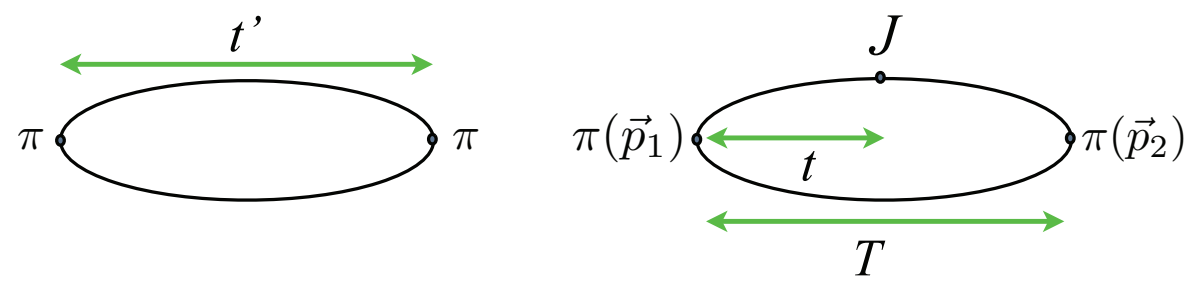

Figure 2: 2 pt and 3 pt correlation functions. $J$ denotes the current, $p_{1}$ is the momentum of the initial pion and $p_{2}$ is the momentum of the pion in the final state. Three different values of $T$, the separation between the initial and final state mesons, were used in this study.

vector current (in the staggered formulation we need a 1-link operator to make a taste singlet, as both pions are Goldstone mesons). A phase at the boundary (twisted boundary condition, [6]) is used to give the quarks momentum: a twist

$$
\Phi\left(x+\hat{e}_{j} L\right)=\mathrm{e}^{i 2 \pi \theta_{j}} \Phi(x)
$$

is equivalent to the quark having a momentum

$$
p_{j}=\frac{2 \pi \theta_{j}}{L} .
$$

$\theta$ can be tuned to get the desired $q^{2}$, the four-momentum transfer defined as

$$
q^{2}=\left(E\left(\vec{p}_{2}\right)-E\left(\vec{p}_{1}\right)\right)^{2}-\left(\vec{p}_{2}-\vec{p}_{1}\right) \cdot\left(\vec{p}_{2}-\vec{p}_{1}\right) .
$$

We calculate the form factor $f_{+}\left(q^{2}\right)$ in the space-like (negative) region of $q^{2}$ near zero (this is the range where experimental data is available). In addition to the 3-point correlators we also need the amplitudes (and energies) from the meson 2-point correlators.

\section{Fitting the correlators}

We fit the 2-point and 3-point correlators simultaneously. We use multi-exponential fits to reduce systematic errors from the excited states, varying the number of exponentials. We take a fit that gives a good $\chi^{2}$ and check that increasing the number of exponentials does not change the result. The preliminary results presented in this paper are from a 5 exponential fit. Bayesian priors 
are used to constrain the fit parameters. We fit all $q^{2}$ values simultaneously to take into account the correlations. The fit functions for the 2-point correlators are of the form

$$
C_{2 p t}(p, t)=\sum_{i} b_{i}^{2}(\vec{p}) \operatorname{fn}\left(E_{i}(\vec{p}), t\right)+\sum_{i} b_{i}^{\prime 2}(\vec{p}) \operatorname{fo}\left(E_{i}^{\prime}(\vec{p}), t\right)
$$

with

$$
\begin{aligned}
& \operatorname{fn}(E, t)=\mathrm{e}^{-E t}+\mathrm{e}^{-E\left(L_{t}-t\right)}, \\
& \text { fo }(E, t)=(-1)^{t / a}\left[\mathrm{e}^{-E t}+\mathrm{e}^{-E\left(L_{t}-t\right)}\right] .
\end{aligned}
$$

Here "fn" are the "normal" states and "fo" are the opposite parity states that appear due to staggered quark formulation. The 3-point correlators are fitted with

$$
\begin{aligned}
C_{3 p t}\left(\vec{p}_{1}, \vec{p}_{2}, t, T\right) & =\sum_{i, j} b_{i}\left(\vec{p}_{1}\right) \operatorname{fn}\left(E_{i}\left(\vec{p}_{1}\right), t\right) J_{i, j}^{n n}\left(\vec{p}_{1}, \vec{p}_{2}\right) b_{j}\left(\vec{p}_{2}\right) \operatorname{fn}\left(E_{j}\left(\vec{p}_{2}\right), T-t\right) \\
& -\sum_{i, j} b_{i}\left(\vec{p}_{1}\right) \operatorname{fn}\left(E_{i}\left(\vec{p}_{1}\right), t\right) J_{i, j}^{n o}\left(\vec{p}_{1}, \vec{p}_{2}\right) b_{j}^{\prime}\left(\vec{p}_{2}\right) \operatorname{fo}\left(E_{j}^{\prime}\left(\vec{p}_{2}\right), T-t\right)+(n \leftrightarrow o) .
\end{aligned}
$$

Note that the amplitudes $b_{i}$ and the energies $E_{i}, E_{i}^{\prime}$ are the same as in the 2-point correlators.

\section{Vector form factor}

The matrix element relevant for the pion form factor is

$$
\left\langle\pi\left(\vec{p}_{1}\right)|J| \pi\left(\vec{p}_{2}\right)\right\rangle=Z \sqrt{4 E_{0}\left(\vec{p}_{1}\right) E_{0}\left(\vec{p}_{2}\right)} J_{0,0}\left(\vec{p}_{1}, \vec{p}_{2}\right),
$$

where $J_{0,0}$ is the ground state amplitude of the 3-point correlator. The form factor $f_{+}$is related to the matrix element as

$$
\left\langle\pi\left(\vec{p}_{1}\right)\left|V_{i}\right| \pi\left(\vec{p}_{2}\right)\right\rangle=f_{+}\left(q^{2}\right)\left(\vec{p}_{1}+\vec{p}_{2}\right)_{i} .
$$

A renormalisation constant $Z$ is needed for the vector current: we normalise the current by demanding that $f_{+}(0)=1$. Our results for the form factor as a function of $q^{2}$ for different lattice ensembles are shown in Fig. 3 along with the experimental data points.

\section{Continuum extrapolation}

We do a simultaneous extrapolation to zero lattice spacing and physical pion mass by fitting the results from the very coarse, coarse and fine lattice to the pole form

$$
f\left(q^{2}\right)=\frac{1}{1-q^{2}\left\langle r^{2}\right\rangle / 6}
$$

allowing for $a^{2}$ and $m_{\pi}$ dependence:

$$
\left\langle r^{2}\right\rangle=A\left(1+B a^{2}+C a^{4}\right)+c_{J} \ln \left(m_{\pi}^{2} / \mu^{2}\right),
$$

where $A, B, C$ are fit parameters and $c_{J}=1 /\left(8 \pi^{2} F^{2}\right)$ is a fixed constant from NLO chiral perturbation theory (here $F$ is the pion decay constant in the chiral limit). The form factor extrapolated to 


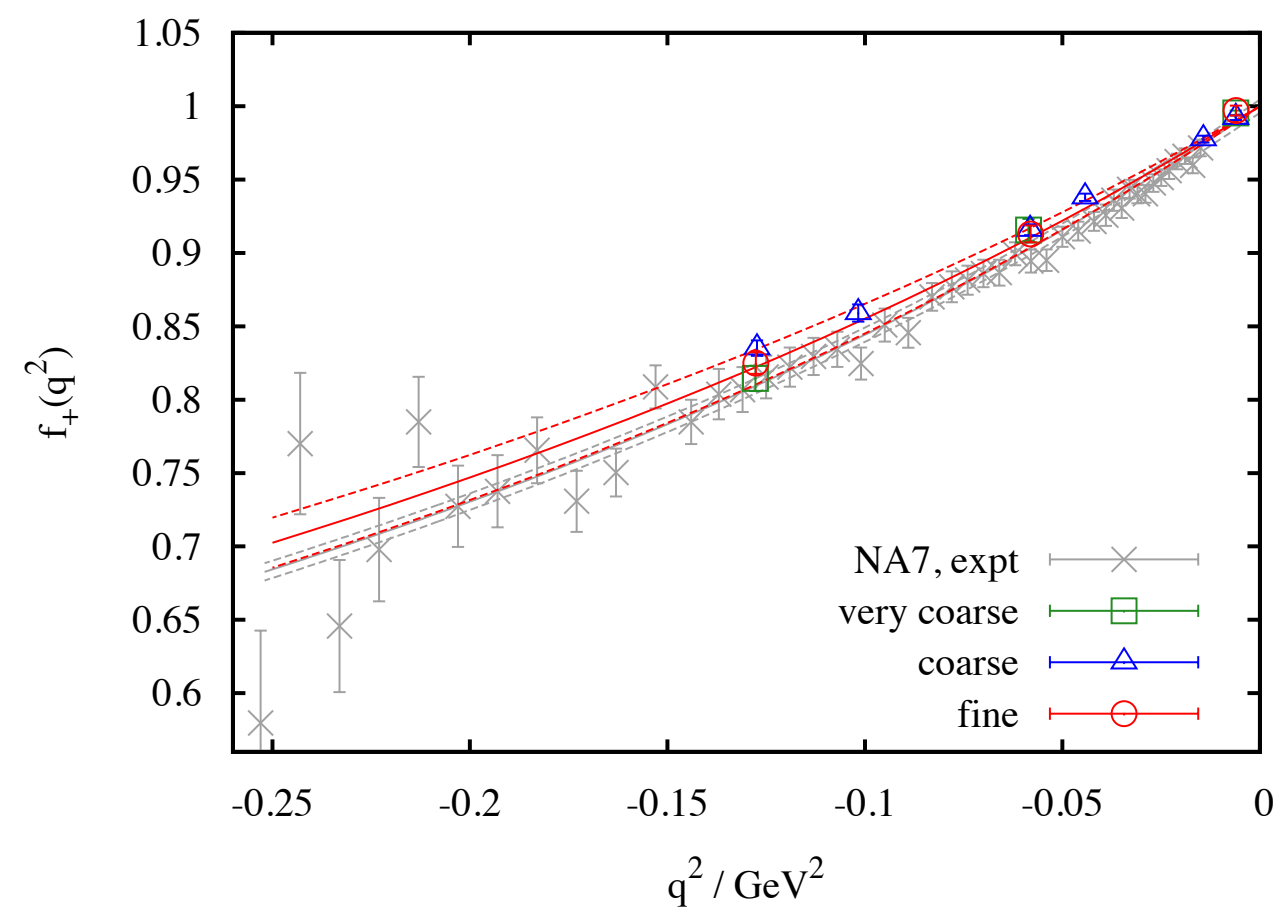

Figure 3: Shape of the pion electromagnetic form factor. Experimental results by NA7 Collaboration are from [3], lattice results by HPQCD from this work. The lines with error bands are from fits to experimental data (grey colour, fit from [3]) and to our lattice results (red colour, this work).

real world is then the $a=0$, physical $m_{\pi}$ part of the fit function, also shown in Fig. 3 with the error bands. The chiral log gives only a very small correction, as the pion masses are very close to the physical value. The slope at $q^{2}=0$ gives the mean square value of the charge radius:

$$
\left\langle r_{v}^{2}\right\rangle=\left.6 \frac{\mathrm{d} f_{+}\left(q^{2}\right)}{\mathrm{d} q^{2}}\right|_{q^{2}=0}
$$

Our preliminary result is $\left\langle r_{v}^{2}\right\rangle=0.40(3) \mathrm{fm}^{2}$. Comparison to other lattice calculations and experiment in Fig. 4 shows good agreement.

The form factor $f_{+}\left(q^{2}\right)$ can be viewed as a Fourier transform of the electric charge distribution. Hence the charge density can be calculated from the vector form factor once its functional form is known. In the non-relativistic limit the charge density is

$$
\rho(R)=\frac{3}{2 \pi R\left\langle r_{v}^{2}\right\rangle} \exp \left(-\frac{R}{\sqrt{\left\langle r_{v}^{2}\right\rangle / 6}}\right)
$$

Using our result $\left\langle r_{v}^{2}\right\rangle=0.40 \mathrm{fm}^{2}$ gives the charge density plotted in Fig. 5 .

\section{Summary}

We have presented here preliminary results from a full Lattice QCD calculation of the pion vector electromagnetic form factor at physical pion mass. The use of twisted boundary conditions 


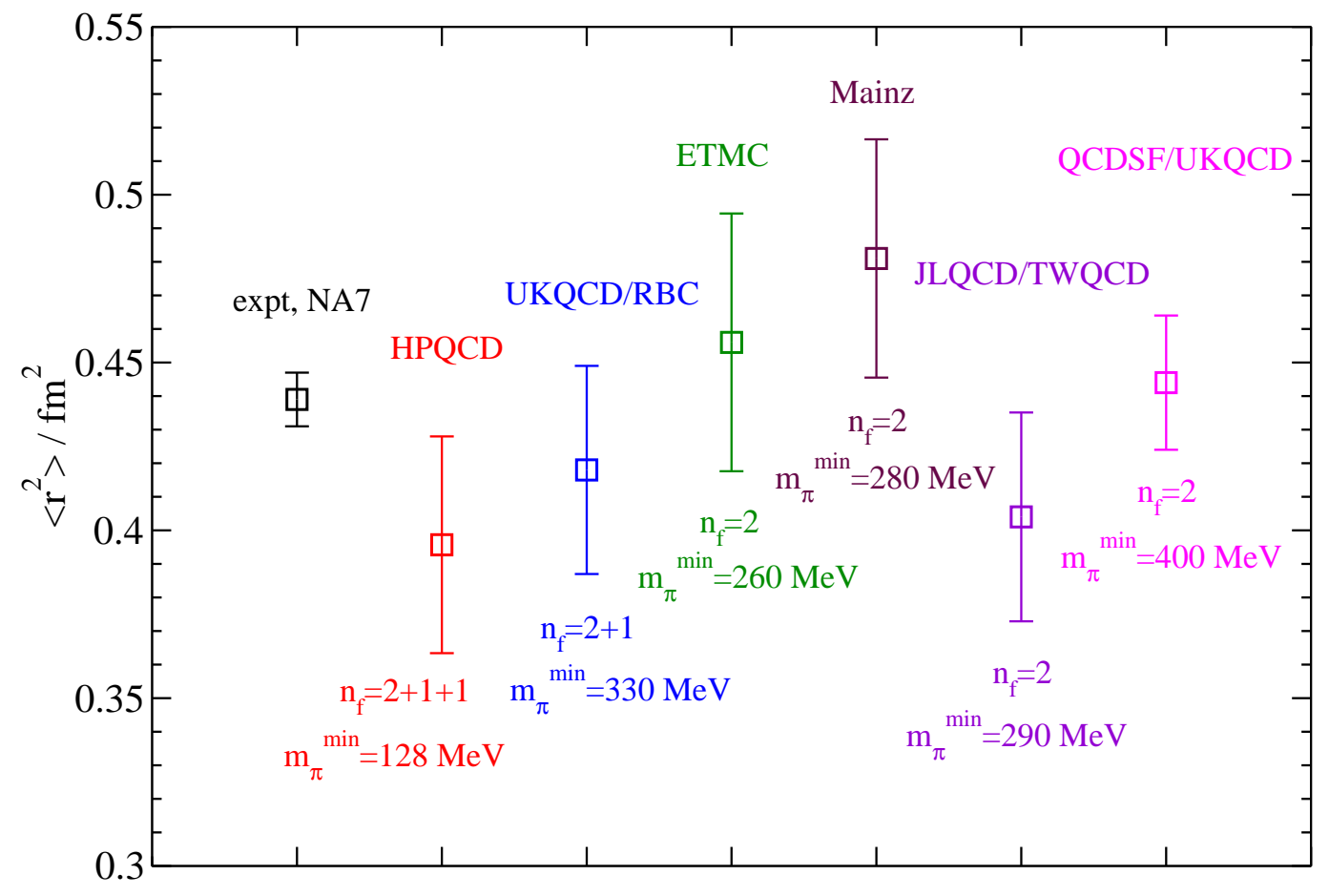

Figure 4: Vector mean square radius. The experimental result is from [3], and the HPQCD result is from this work. Other lattice results are from $[7,1,8,9,10]$ (from left to right). $n_{f}$ is the number of flavors and $m_{\pi}^{\min }$ is the smallest pion mass used in that calculation. The results shown here are each group's final result after continuum and chiral extrapolation.

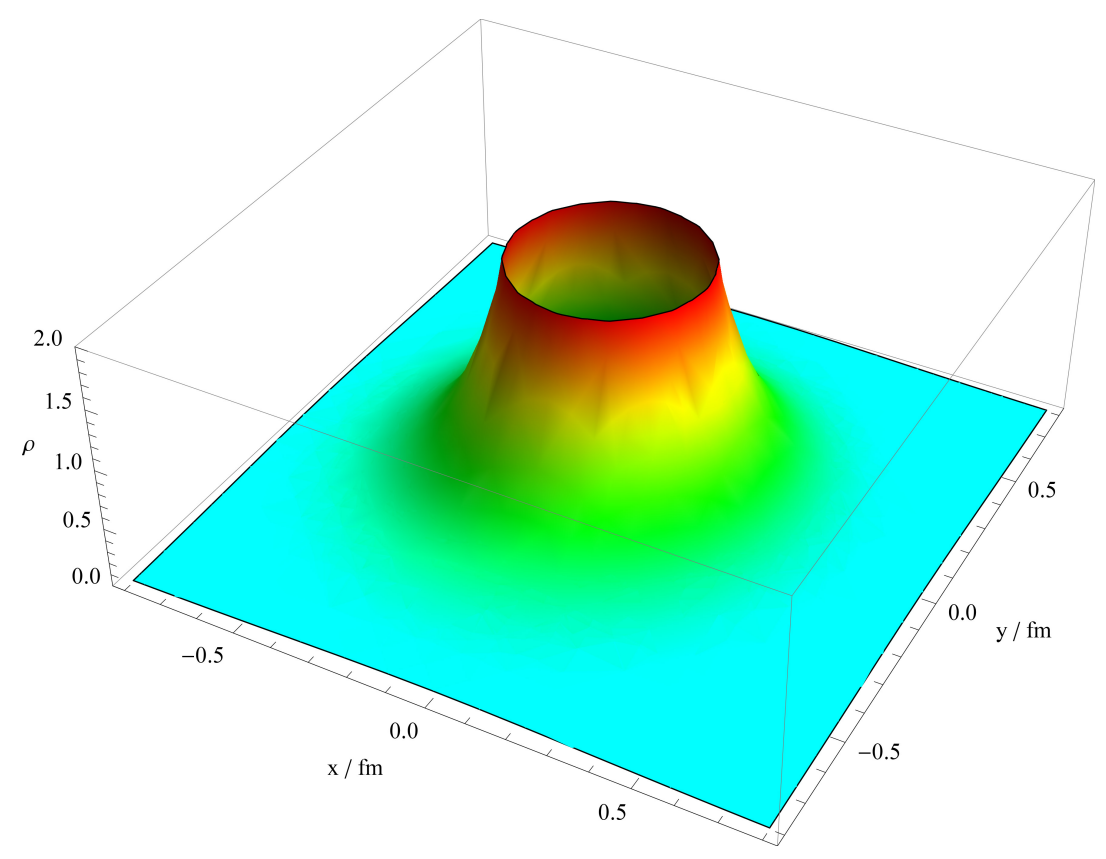

Figure 5: Non-relativistic charge density calculated using the pole form and $\left\langle r_{v}^{2}\right\rangle$ from our fit. We omit $z$ direction here for clarity and plot the charge density against $x$ and $y$. 
allows us to calculate the form factor in the $q^{2}$ range from 0 to $-0.12 \mathrm{GeV}^{2}$ where experimental data is available. We also determine the charge radius: our preliminary result is $\left\langle r_{v}^{2}\right\rangle=0.40(3) \mathrm{fm}^{2}$. Comparison with experiment shows very good agreement.

\section{Acknowledgements}

We are grateful to MILC for the use of their gauge configurations and the MILC code. We used the Darwin Supercomputer in Cambridge as part of the DiRAC facility jointly funded by STFC, BIS and the Universities of Cambridge and Glasgow.

\section{References}

[1] ETM Collaboration, R. Frezzotti, V. Lubicz, and S. Simula, PRD 79 (2009) 074506

[2] B.B. Brandt, arXiv 1310.6389 [hep-lat]

[3] NA7 Collaboration, S.R. Amendolia et al., Nucl. Phys. B277 (1986) 168

[4] MILC Collaboration, A. Bazavov, C. Bernard, C. DeTar, W. Freeman, S. Gottlieb, U. M. Heller, J. E. Hetrick, J. Laiho, L. Levkova, M. Oktay, J. Osborn, R.L. Sugar, D. Toussaint, R.S. Van de Water, PRD 82 (2010) 074501, arXiv:1004.0342; and A. Bazavov, C. Bernard, C. DeTar, W. Freeman, S. Gottlieb, U. M. Heller, J. E. Hetrick, J. Komijani, J. Laiho, L. Levkova, J. Osborn, R. L. Sugar, D. Toussaint, R. S. Van de Water, R. Zhou, PRD 87 (2013) 054505, arXiv:1212.4768

[5] HPQCD Collaboration, R. J. Dowdall, C. T. H. Davies, G. P. Lepage, C. McNeile, PRD 88 (2013) 074504, arXiv:1303.1670

[6] D. Guadagnoli, F. Mescia, and S. Simula, PRD 73 (2006) 114504

[7] UKQCD and RBC Collaborations, P.A. Boyle, J.M. Flynn, A. Jüttner, C. Kelly, H. Pedroso de Lima, C.M. Maynard, C.T. Sachrajda, and J.M. Zanotti, JHEP 07 (2008) 112

[8] B.B. Brandt, A. Jüttner, H. Wittig, arXiv 1306.2916 [hep-lat],

V. Gülpers, G. von Hippel and H. Wittig, PoS (Lattice 2012) 181 and arXiv 1309.2104 [hep-lat]

[9] JLQCD and TWQCD Collaborations, T. Kaneko, S. Aoki, G. Cossu, X. Feng, H. Fukaya, S. Hashimoto, J. Noaki, and T. Onogi,

PoS (Lattice 2008) 158

[10] QCDSF and UKQCD Collaborations, D. Brömmel et al., Eur. Phys. J. C51 (2007) 335 